Supplement of Biogeosciences, 16, 1411-1432, 2019

https://doi.org/10.5194/bg-16-1411-2019-supplement

(C) Author(s) 2019. This work is distributed under

the Creative Commons Attribution 4.0 License.

(c) (1)

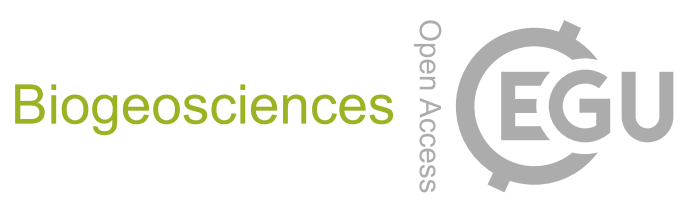

Supplement of

\title{
Dissolved organic matter characteristics of deciduous and coniferous forests with variable management: different at the source, aligned in the soil
}

Lisa Thieme et al.

Correspondence to: Lisa Thieme (1.thieme@campus.tu-berlin.de)

The copyright of individual parts of the supplement might differ from the CC BY 4.0 License. 
Table S1: Site and plot description.

\begin{tabular}{|c|c|c|c|c|c|c|c|c|c|}
\hline & \multicolumn{3}{|c|}{ Schwäbische Alb (ALB) } & \multicolumn{3}{|c|}{ Hainich-Dün (HAI) } & \multicolumn{3}{|c|}{ Schorfheide-Chorin (SCH) } \\
\hline \multicolumn{10}{|l|}{ site description } \\
\hline Location & \multicolumn{3}{|c|}{ Southwest Germany $\left(53^{\circ} 2^{\prime} \mathrm{N}, 13^{\circ} 51^{`} \mathrm{E}\right)$} & \multicolumn{3}{|c|}{ Central Germany $\left(51^{\circ} 10^{\prime} \mathrm{N}, 10^{\circ} 23^{\prime} \mathrm{E}\right)$} & \multicolumn{3}{|c|}{ Northeast Germany $\left(53^{\circ} 2^{\prime} \mathrm{N}, 13^{\circ} 51^{\prime} \mathrm{E}\right)$} \\
\hline Altitude & \multicolumn{3}{|c|}{$460-860 \mathrm{~m}$ a.s.l. } & \multicolumn{3}{|c|}{285 - $550 \mathrm{~m}$ a.s.l. } & \multicolumn{3}{|c|}{ 3-140 $m$ a.s.l. } \\
\hline Mean anual temperature & \multicolumn{3}{|c|}{$6-7^{\circ} \mathrm{C}$} & \multicolumn{3}{|c|}{$6.5-8^{\circ} \mathrm{C}$} & \multicolumn{3}{|c|}{$8-8.5^{\circ} \mathrm{C}$} \\
\hline Mean anual precipitation & \multicolumn{3}{|c|}{$700-1000 \mathrm{~mm}$} & \multicolumn{3}{|c|}{$500-800 \mathrm{~mm}$} & \multicolumn{3}{|c|}{$500-600 \mathrm{~mm}$} \\
\hline Bedrock & \multicolumn{3}{|c|}{ Jurassic limestone } & \multicolumn{3}{|c|}{ Triassic limestone with loess cover } & \multicolumn{3}{|c|}{ Quartzitic glacial till } \\
\hline Main soil types & \multicolumn{3}{|c|}{ Leptosols, Cambisols } & \multicolumn{3}{|c|}{ Luvisols, Stagnosols } & \multicolumn{3}{|c|}{ Cambisols, Albeluvisols } \\
\hline Main tree species & \multicolumn{3}{|c|}{$\begin{array}{c}\text { Fagus sy/vatica L. } \\
\text { Picea abies (L.) H. Karst. }\end{array}$} & \multicolumn{3}{|c|}{$\begin{array}{l}\text { Fagus sy/vatica L. } \\
\text { Picea abies (L.) H. Karst. } \\
\text { Fraxinus exelsior L. }\end{array}$} & \multicolumn{3}{|c|}{$\begin{array}{l}\text { Fagus sylvatica } \mathrm{L} \text {. } \\
\text { Pinus sylvestris L. } \\
\text { Quercus spp. }\end{array}$} \\
\hline \multicolumn{10}{|l|}{ plot description } \\
\hline Plot ID & AEW1-AEW3 & AEW4 - AEW6 & AEW7 - AWE9 & HEW1 - HWE3 & HEW4 - HEW6 & HEW10 - HEW12 & SEW1 - SEW3 & SEW5 - SEW6 & SEW7 - SEW9 \\
\hline Main tree species & spruce & beech & beech & spruce & beech & Beech & pine & beech & beech \\
\hline Management category & coniferous age-class & deciduous age-class & unmanaged & coniferous age-class & deciduous age-class & unmanaged & coniferous age-class & deciduous age-class & unmanaged \\
\hline Stand density ( $n$ ha-1) & $480-752$ & $104-2219$ & $312-444$ & $320-720$ & thicket; $488 ; 300$ & $260-564$ & $444-1440$ & 92 , thicket & $168-284$ \\
\hline Mean $d b h(\mathrm{~cm})$ & $26.3-32.2$ & $12.4-45.2$ & $27.1-36.4$ & $25.2-40.2$ & NA, 24.7, 38.7 & $22.8-35.0$ & $17.3-33.1$ & $57.2, \mathrm{NA}$ & $40.7-51.0$ \\
\hline Basal area $\left(m^{2} h a-1\right)$ & $44.0-44.5$ & $18.0-28.7$ & $31.4-46.9$ & $28.3-42.4$ & NA, 28.3, 39.5 & $35.4-39.3$ & $36.4-39.9$ & 28.2, NA & $39.3-43.4$ \\
\hline ForMI & $1.8052-2.3536$ & $0.9392-1.5632$ & $0.0000-1.0285$ & $1.3073-2.1979$ & $1.890,0.9556,0.7467$ & $0.0000-0.5177$ & $1.4052-1.9003$ & $0.6890,1.4018$ & $0.0855-0.6459$ \\
\hline
\end{tabular}


Table S2:Sample information. DOM characterization: fluorescence. $T F=$ Throughfall, $S F=$ Stemflow, $L L=L i t t e r$ Leachate, TOP $=$ Topsoil solution, $\mathrm{SUB}=$ Subsoil Solution .

\begin{tabular}{|c|c|c|c|c|c|c|c|}
\hline \multirow[t]{2}{*}{ site } & \multirow[t]{2}{*}{ management category } & \multirow[t]{2}{*}{ plot ID } & \multicolumn{5}{|c|}{ sample number per ecosystem flux } \\
\hline & & & TF & SF & $\mathrm{LL}$ & TOP & SUB \\
\hline \multirow[t]{9}{*}{ Hainich Dün } & coniferous age-class & HEW1 & 3 & 1 & 2 & 2 & 2 \\
\hline & & HEW2 & 3 & 2 & 2 & 2 & 2 \\
\hline & & HEW3 & 2 & 2 & 2 & 1 & 2 \\
\hline & deciduous age-class & HEW4 & 1 & NA & 2 & NA & NA \\
\hline & & HEW5 & 2 & 3 & 2 & 2 & 2 \\
\hline & & HEW6 & 3 & 3 & 2 & 2 & 2 \\
\hline & beech unmanaged & HEW10 & 2 & 3 & 2 & 2 & NA \\
\hline & & HEW11 & 3 & 2 & 2 & 2 & 2 \\
\hline & & HEW12 & 3 & 1 & 3 & 2 & 2 \\
\hline \multirow[t]{8}{*}{ Schorfheide Chorin } & coniferous age-class & SEW1 & 10 & 10 & 15 & 1 & 6 \\
\hline & & SEW2 & 12 & 13 & 12 & NA & 2 \\
\hline & & SEW3 & 12 & 9 & 13 & 2 & 2 \\
\hline & deciduous age-class & SEW5 & 10 & 13 & 9 & 3 & 1 \\
\hline & & SEW6 & 11 & 13 & 16 & 3 & 7 \\
\hline & beech unmanaged & SEW7 & 10 & 11 & 13 & 4 & NA \\
\hline & & SER8 & 10 & 15 & 9 & 3 & 4 \\
\hline & & SEW9 & 12 & 11 & 9 & 3 & 3 \\
\hline
\end{tabular}

Table S3: Sample information. DOM characterization: FTICR-MS. TF $=$ Throughfall, SF $=$ Stemflow, LL $=$ Litter Leachate, TOP $=$ Topsoil solution, SUB $=$ Subsoil Solution.

\begin{tabular}{lccccc}
\hline site & management category & \multicolumn{4}{c}{ plot composition per ecosystem flux } \\
& & TF & SF & LL & SUB \\
\hline Schorfheide-Chorin & coniferous age-class & SEW1+3 & SEW1+3 & SEW1+3 & SEW1+3 \\
& beech unmanaged & SEW8+9 & SEW8+9 & SEW8+9 & SEW8+9
\end{tabular}

Table S4: Sample information. DOM biodegradability. TF = Throughfall, SF = Stemflow, LL = Litter Leachate, TOP $=$ Topsoil solution, SUB $=$ Subsoil Solution .

\begin{tabular}{|c|c|c|c|c|}
\hline \multirow[t]{2}{*}{ site } & \multirow[t]{2}{*}{ management category } & \multicolumn{3}{|c|}{ plot composition per ecosystem flux } \\
\hline & & TF & SF & LL \\
\hline \multirow[t]{3}{*}{ Schwäbische Alb } & coniferous age-class & AEW1-3 & NA & AEW1-3 \\
\hline & deciduous age-class & AEW4-6 & AEW4-6 & AEW4-6 \\
\hline & beech unmanaged & AEW7-9 & AEW7-9 & NA \\
\hline \multirow[t]{3}{*}{ Hainich Dün } & coniferous age-class & HEW1-3 & HEW1-3 & HEW1+2 \\
\hline & deciduous age-class & HEW5+6 & HEW5+6 & HEW5+6 \\
\hline & beech unmanaged & HWE 10-12 & HWE10-12 & HWE10-12 \\
\hline \multirow[t]{3}{*}{ Schorfheide Chorin } & coniferous age-class & SEW1-3 & SEW1-3 & SEW1-3 \\
\hline & deciduous age-class & SEW4+5 & SEW4+5 & SEW4+5 \\
\hline & beech unmanaged & SEW6-9 & SEW6-9 & SEW6-9 \\
\hline
\end{tabular}


Table S5: Calculated degradation rate constants $(k)$ and relative amount of biodegradable dissolved organic carbon (BDOC) for different ecosystem fluxes in the Schwäbische Alb (ALB), the Hainich Dün (HAI) and the Schorfheide Chorin (SCH) sites. $\mathrm{K}$ was calculated using a two parameter single exponential model.

\begin{tabular}{|c|c|c|c|c|c|}
\hline & site & management category & $k\left[d^{-1}\right]$ & St. Error & $\mathrm{BDOC}[\%]$ \\
\hline \multirow[t]{9}{*}{ throughfall } & $\mathrm{SCH}$ & coniferous age-class & 0.0063 & 0.0008 & 17 \\
\hline & ALB & coniferous age-class & 0.0081 & 0.0013 & 18 \\
\hline & $\mathrm{HAl}$ & deciduous age-class & 0.0082 & 0.0011 & 19 \\
\hline & ALB & unmanaged & 0.0116 & 0.0017 & 23 \\
\hline & ALB & deciduous age-class & 0.0106 & 0.0017 & 24 \\
\hline & $\mathrm{HAl}$ & coniferous age-class & 0.0098 & 0.0019 & 27 \\
\hline & $\mathrm{SCH}$ & deciduous age-class & 0.0136 & 0.0023 & 29 \\
\hline & $\mathrm{SCH}$ & unmanaged & 0.0154 & 0.0009 & 34 \\
\hline & $\mathrm{HAl}$ & unmanaged & 0.0148 & 0.0032 & 36 \\
\hline \multirow[t]{8}{*}{ stemflow } & $\mathrm{HAl}$ & deciduous age-class & 0.0052 & 0.0015 & 15 \\
\hline & $\mathrm{SCH}$ & coniferous age-class & 0.0084 & 0.0011 & 18 \\
\hline & $\mathrm{HAl}$ & unmanaged & 0.0116 & 0.0013 & 30 \\
\hline & $\mathrm{SCH}$ & unmanaged & 0.0122 & 0.0018 & 30 \\
\hline & ALB & deciduous age-class & 0.0179 & 0.0030 & 32 \\
\hline & $\mathrm{SCH}$ & deciduous age-class & 0.0183 & 0.0012 & 38 \\
\hline & $\mathrm{HAl}$ & coniferous age-class & 0.0175 & 0.0016 & 40 \\
\hline & ALB & unmanaged & 0.0213 & 0.0021 & 40 \\
\hline \multirow[t]{8}{*}{ litter leachate } & ALB & coniferous age-class & 0.0040 & 0.0012 & 8 \\
\hline & $\mathrm{SCH}$ & coniferous age-class & 0.0047 & 0.0011 & 10 \\
\hline & $\mathrm{SCH}$ & unmanaged & 0.0050 & 0.0011 & 11 \\
\hline & $\mathrm{SCH}$ & deciduous age-class & 0.0049 & 0.0012 & 11 \\
\hline & ALB & deciduous age-class & 0.0070 & 0.0021 & 12 \\
\hline & $\mathrm{HAl}$ & coniferous age-class & 0.0052 & 0.0018 & 17 \\
\hline & $\mathrm{HAl}$ & unmanaged & 0.0057 & 0.0012 & 17 \\
\hline & $\mathrm{HAl}$ & deciduous age-class & 0.0059 & 0.0011 & 18 \\
\hline
\end{tabular}


Table S6: sample information: DOM biodegradability. Mean concentrations of nitrogen and phosphor in samples before incubation. $\mathrm{NH}_{4}-\mathrm{N}+\mathrm{NO}_{3}-\mathrm{N}=$ concentration of ammonium and nitrate nitrogen, $\mathrm{PO}_{4}-\mathrm{P}=$ concentration of ortho phophate

\begin{tabular}{|c|c|c|c|c|c|c|}
\hline & \multicolumn{2}{|l|}{ TF } & \multicolumn{2}{|l|}{ SF } & \multicolumn{2}{|l|}{ LL } \\
\hline & $\begin{array}{c}\mathrm{NH}_{4}-\mathrm{N}+\mathrm{NO}_{3}-\mathrm{N} \\
{\left[\mathrm{mg} \mathrm{L}^{-1}\right]}\end{array}$ & $\begin{array}{l}\mathrm{PO}_{4}-\mathrm{P} \\
{\left[\mathrm{mg} \mathrm{L}^{-1}\right]}\end{array}$ & $\begin{array}{c}\mathrm{NH}_{4}-\mathrm{N}+\mathrm{NO}_{3}-\mathrm{N} \\
{\left[\mathrm{mg} \mathrm{L}^{-1}\right]}\end{array}$ & $\begin{array}{l}\mathrm{PO}_{4}-\mathrm{P} \\
{\left[\mathrm{mg} \mathrm{L}^{-1}\right]}\end{array}$ & $\begin{array}{c}\mathrm{NH}_{4}-\mathrm{N}+\mathrm{NO}_{3}-\mathrm{N} \\
{\left[\mathrm{mg} \mathrm{L}^{-1}\right]}\end{array}$ & $\begin{array}{c}\mathrm{PO}_{4}-\mathrm{P} \\
{\left[\mathrm{mg} \mathrm{L}^{-1}\right]}\end{array}$ \\
\hline \multicolumn{7}{|l|}{ Schwäbische Alb } \\
\hline coniferous age-class & 1.42 & 0.047 & & & 3.85 & 0.206 \\
\hline deciduous age-class & 1.02 & 0.144 & 4.37 & 0.830 & 0.82 & 0.184 \\
\hline unmanaged & 1.45 & 0.052 & 3.44 & 0.136 & 5.14 & 0.349 \\
\hline \multicolumn{7}{|l|}{ Hainich Dün } \\
\hline coniferous age-class & 7.39 & 0.330 & 18.68 & 0.086 & 4.95 & 0.113 \\
\hline deciduous age-class & 2.97 & 0.146 & 2.24 & 0.008 & 5.23 & 0.828 \\
\hline unmanaged & 2.44 & 0.124 & 2.19 & 0.017 & 7.17 & 1.158 \\
\hline \multicolumn{7}{|l|}{ Schorfheide Chorin } \\
\hline coniferous age-class & 0.92 & 0.018 & 6.57 & 0.015 & 0.77 & 0.301 \\
\hline deciduous age-class & 0.71 & 0.232 & 1.34 & 0.124 & 7.04 & 0.323 \\
\hline unmanaged & 2.27 & 0.727 & 1.25 & 0.130 & 1.97 & 0.504 \\
\hline
\end{tabular}




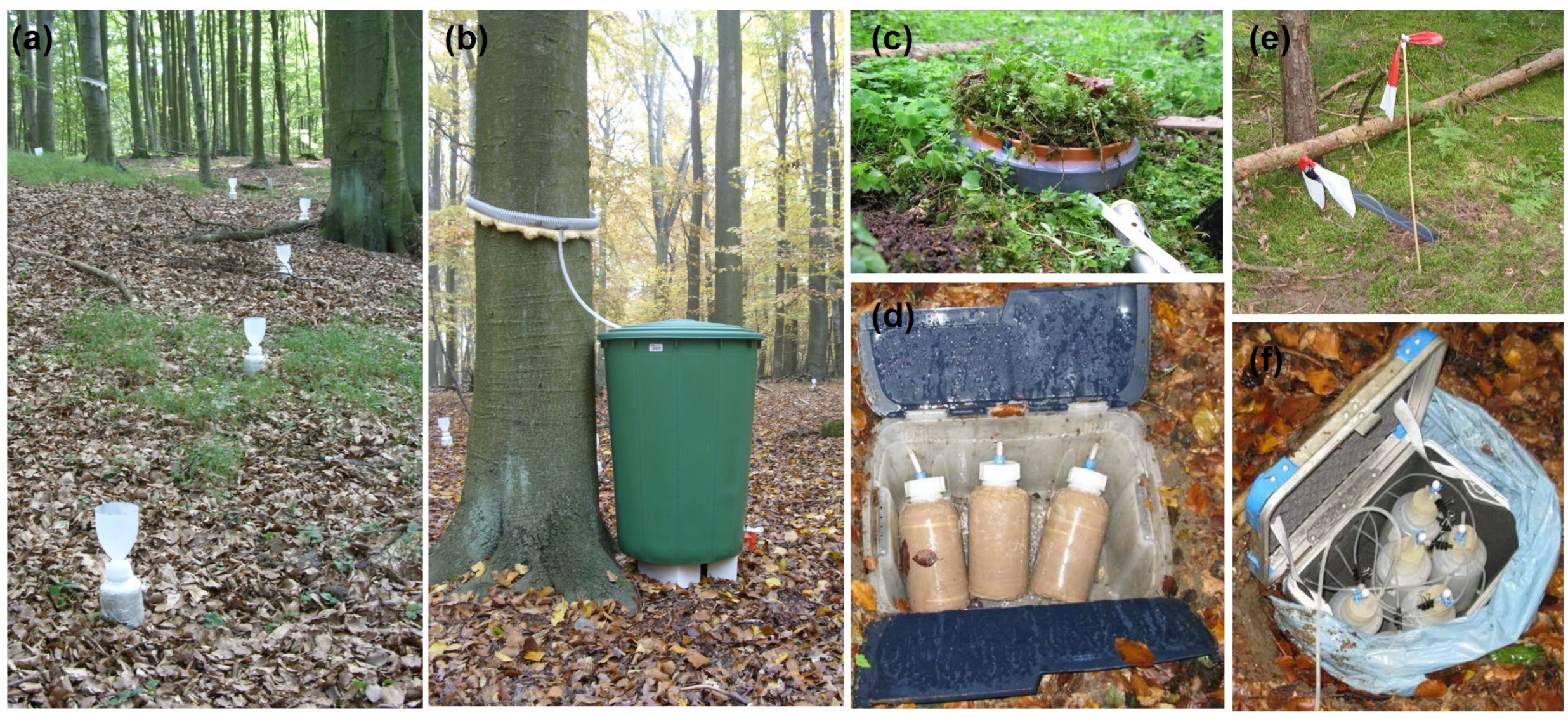

Figure S1: Fotos of sampling installations on forest plots. (a) Throughfall (TF) collection bottels, (b) Stemflow (SF) collection collar on beech tree, connected with a 200 L barrel, (c) Litter Leachate (LL) collector and (d) collection bottles (free draining), (e) aboveground view of suction cubs for Soil Solution (TOP and SUB) sampling and (f) collection bottles (manual applied preassure). 

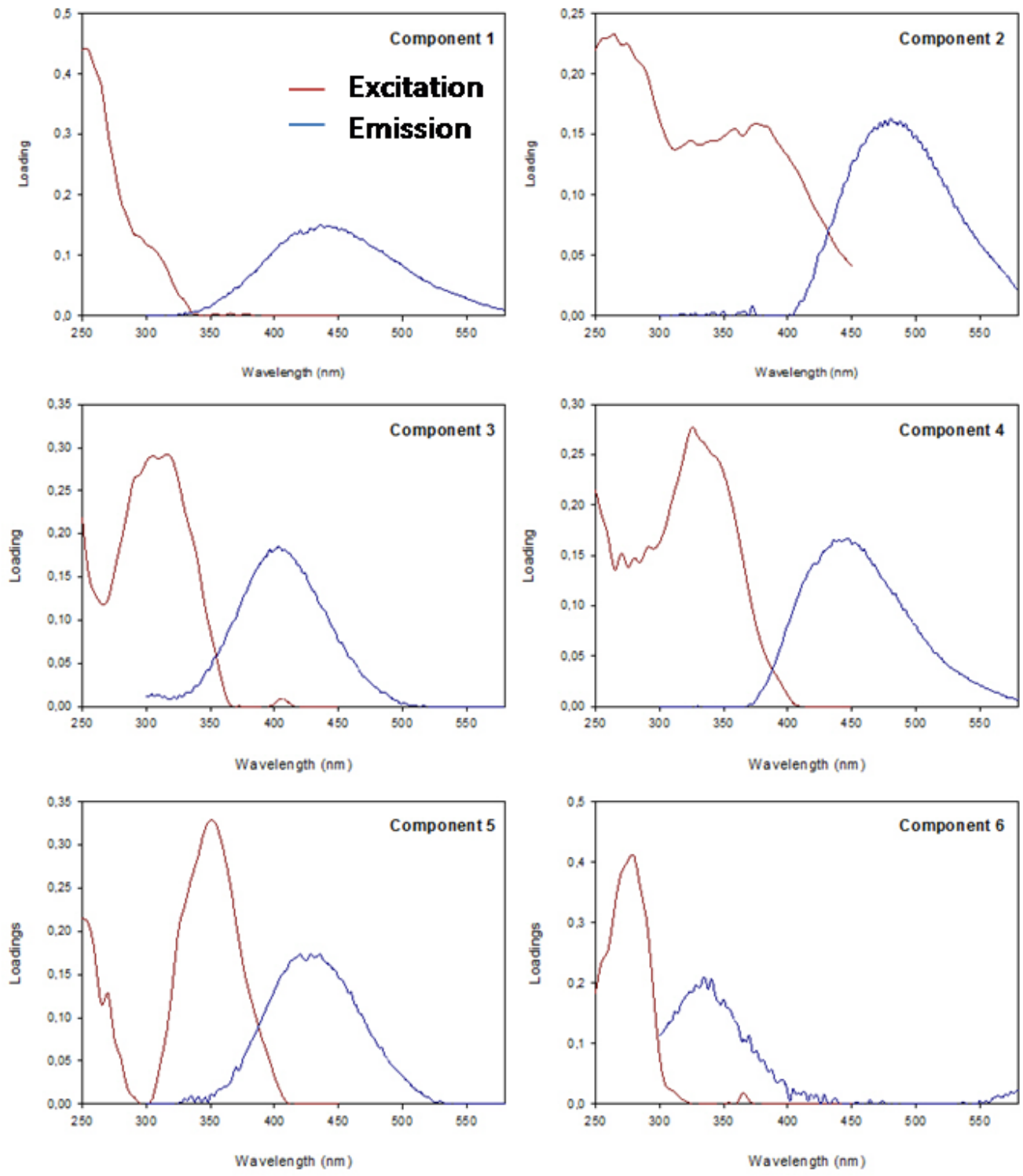

Figure S2: Excitation (red) and emission (blue) spectra of PARAFAC components (C1-C6). 